\title{
EVALUACIÓN DE LA MELAZA DE CAÑA COMO SUSTRATO PARA EL CRECIMIENTO DE Lactobacillus plantarum
}

\section{EVALUATION OF CANE MOLASSES AS SUBSTRATE FOR Lactobacillus plantarum GROWTH}

\author{
Juliana Andrea Ossaํ, María Consuelo Vanegas², Ángela María Badillo³
}

\begin{abstract}
${ }^{1}$ Microbióloga Agrícola y Veterinaria. Estudiante de Maestría de Ciencias Biológicas de la Universidad de Los Andes, Laboratorio de Ecología Microbiana y de Alimentos, LEMA. ${ }^{2}$ Microbióloga M.Sc. Profesora asociada, Directora del Laboratorio de Ecología Microbiana y de Alimentos, LEMA, Universidad de Los Andes. mvanegas@uniandes.edu.co. ${ }^{3}$ Microbióloga. Laboratorio de Ecología Microbiana y de Alimentos, LEMA, Universidad de Los Andes. Carrera $1 \mathrm{~N}^{\circ}$ 18A 10J-209. Bogotá-Cundinamarca, Colombia. Correspondencia: ja.ossa907@uniandes.edu.co
\end{abstract}

Rev. U.D.C.A Act. E Div. Cient. 13 (1): 97-104, 2010

\section{RESUMEN}

La importancia de bacterias acido lácticas (BAL), como los Lactobacillus sp. en Colombia y en el mundo, hace necesario encontrar métodos óptimos para su crecimiento, utilizando sustratos diferentes para su desarrollo. Se empleó melaza de caña como sustrato iniciador para el incremento de L. plantarum. Se evaluaron condiciones y variables que afectan el crecimiento microbiano. Se manejó como cepa control $L$. plantarum WS417 y se inocularon diferentes concentraciones de melaza estéril a diferentes valores de $\mathrm{pH}$, temperatura y agitación. El crecimiento, se determinó con microbiología tradicional utilizando recuentos directos en placa, con agar MRS. La interpretación de resultados, se realizó con un análisis de varianza factorial, observando interacciones de las variables, respecto al recuento microbiológico. Las condiciones óptimas para el crecimiento de $L$. plantarum fueron $20 \%$ concentración, $30 \pm 1^{\circ} \mathrm{C}$ durante 24 horas, $5,2 \pm 0,1(\mathrm{pH})$ y $100 \mathrm{rpm}$, donde se obtuvo un recuento de $43 \times 10^{\circ} \mathrm{UFC} /$ $\mathrm{mL}$. Se concluyó que la melaza de caña podría ser usada como sustrato para el desarrollo de Lactobacillus sp.

Palabras clave: Melaza de caña, sustrato, bacterias ácido lácticas.

\section{SUMMARY}

Due the importance of lactic acid bacteria (LAB) in Colombia and worldwide, it has been necessary to optimize the culture conditions using different kind of substrates to increase growth. In this research, cane molasses was exploited as an indicator substrate to determinate Lactobacillus plantarum growth. Different conditions and variables that affect the microbial development were evaluated. L. plantarum (WS417) was used as a control strain, inoculating diverse sterile molasses concentrations at different $\mathrm{pH}$ values, temperature and agitation. The strain growth was evaluated by traditional microbiology, direct bacteria count, seeded in MRS agar plates. Relationships between biomass and effectiveness of different culture conditions were estimated by factorial variance analysis, showing the results as optimal growth conditions the content of $20 \%$ cane molasses, $30 \pm 1^{\circ} \mathrm{C}$ for 24 hours, $\mathrm{pH} 5,2 \pm 0,1$, and $100 \mathrm{rpm}$ with counts of $43 \times 10^{9} \mathrm{UFC} / \mathrm{mL}$. It was concluded that cane molasses could be employed as growth substrate for Lactobacillus sp.

Key words: Cane molasses, substrate, lactic acid bacteria. 


\section{INTRODUCCIÓN}

Los Lactobacillus spp. son bacterias ácido lácticas (BAL) que se caracterizan por los diferentes usos e importancia a nivel industrial y, en ocasiones, utilizadas como fermentadores de alimentos cárnicos, lácteos y vegetales, además del uso en biopreservación, para incrementar la vida útil de los productos o como potencial probiótico en la industria. Desde el siglo pasado estos microorganismos han demostrado múltiples efectos positivas en la salud de animales y del hombre (Silveira et al. 2003; Cabeza, 2006; Vásquez et al. 2009; Gálvez et al. 2007; Castellano et al. 2008; Fiorentini et al. 2001).

Los probióticos tienen diferentes efectos en el ser humano, es así que modifican la microflora intestinal, influyen directa e indirectamente en el estado de la salud, a través de producción de vitaminas y ácidos grasos de cadena corta, colaboran con la degradación de sustancias alimenticias no digeridas, estimulan la respuesta inmune y dan protección frente a microorganismos enteropatógenos (Lee $\mathcal{E}$ Salminen, 1995; Holzapfel, 2001).

Según, Vargas et al. (2004), cerca del 65\% de los alimentos que participan en el mercado mundial son productos con probióticos y los Lactobacillus son una de las bacterias más empleados en el mercado nacional. Las cepas mundiales consideradas como probióticos y utilizadas como ingredientes del producto, son las siguientes: L. acidophilus, L. bulgaricus, L. lactis, L. casei, L. plantarum, L. rhamnosus, L. reuteri, $L$. paracesei, L. fermentum, L. helveticus, Bifidobacterium, $B$. adolescentis, $B$. angulatum, $B$. bifidum, $B$. breve, $B$. catenulatum, B. dentium, B. infantis, B. longum, $B$. pseudocatenulatum, B. infantis, L. acidophilus NCFM (Rhodia, EE.UU.) L. acidophilus NCFB 1748.L. acidophilus LA1 (al cual se lo renombró como $L$. johnsonii LJ1 -comercializado por Nestlé, Suiza). L. casei Shirota (Yakult, Japón). L. casei CRL431 (Chr. Hansen, EE.UU.). L. fermentum RC-14 (Urex, Canadá). L. casei DN114 (Danone, Francia).L. crispatus CTV05 (Gynelogix, EE.UU.). L. reuteri MM53 (BioGaia, Suecia). L. rhamnosus GG (Valio, Finlandia). L. rhamnosus GR-1 (Urex, Canadá). L. plantarum 299V (producto de Probi, Suecia) (Shah E Lankaputhra, 2002; Mantello, 2007).

En Colombia, las bacterias que se manejan para productos probióticos son cepas importadas de países europeos y Japón, lo que implica costos de adquisición. Adicionalmente, si las empresas comercializadoras de alimentos fermentados (95\%) del mercado nacional, trabajaran con la búsqueda de cultivos y condiciones que proporcionen la obtención de mayores densidades del microorganismo (>107 $\mathrm{UFC} / \mathrm{g})$, generaría un mayor impacto en el desarrollo de estas bacterias, a partir de un medio no láctico para ampliar sus aplicaciones a otros alimentos (Vargas et al. 2004), ya sea para consumo humano o animal, biopreservación, probióticos, entre otros, con la correspondiente reducción de costos para los consumidores, lo cual seria un gran aporte a la industria nacional en la producción de bacterias ácido lácticas.

Actualmente, en Colombia la industria de lácteos es las que más utiliza probióticos (Vargas et al. 2004). La venta de productos probióticos importados es distribuida a un grupo de industrias limitado, debido al costo y al valor comercial, tales como Alpina, Parmalat, Corpoica, entre otras (Castro \& Rodríguez, 2005; www.alpina.com. co). Entre los años 2000 y 2005, se ha incrementado el dinamismo del sector lácteo en un $8,1 \%$ de leche y de un 7,3\% de otros productos lácteos (DANE-Agrocadenas, 2006).

El mercado de alimentos que aprovecha BAL continua creciendo mundialmente y en Colombia es necesario contar con sistemas de producción, de métodos y de medios de cultivos adecuados para dicho objetivo; se genera la búsqueda de condiciones y factores que permitan la producción de BAL, como una necesidad de la industria colombiana, para así poder producir microorganismos iniciadores de alimentos fermentados como cárnicos, yogures, quesos, entre otros, sino, también para ser utilizados como ingredientes o aditivos prebióticos, para aumentar los beneficios del cliente por consumo de dichos productos y, de esta manera dotarles de un valor agregado.

Estudios anteriores muestran que el MRS (Man, Rogosa y Sharpe) es un medio de cultivo adecuado para la recuperación de Lactobacillus sp. en condiciones de laboratori, y su costo se hace elevado para el uso industrial en grandes cantidades (Vargas et al. 2004). En la industria se han evaluado diferentes sustratos para el crecimiento de BAL, como el medio de cultivo agar leche y leche descremada, que favorece el aislamiento de Lactobacillus sp. (Cogan et al. 1997; Simova et al. 2002). Poca es la información disponible de sustratos 
utilizados para el cultivo de bacterias ácido lácticas, a nivel industrial, pero tradicionalmente, se ha utilizado la miel o melaza "blackstrap", que es un líquido denso, viscoso de color oscuro y que contiene sales y otros compuestos solubles en álcali; es un producto final de la fabricación o refinación de la sacarosa, glucosa y fructosa procedente de la caña de azúcar; además, contienen sustancias no fermentables y melanoidinas (a base de nitrógeno), derivados a partir de la condensación del azúcar y aminocompuestos (Honig, 1974; Swan E Karalazos, 1990). Este subproducto es comúnmente destinado para la producción de alimentos concentrados de animales y como suplemento alimenticio para el hombre, a pesar que, en la actualidad su costo depende de otros factores, como el tema de biocombustibles, por ejemplo. Ello hace necesario analizar las condiciones que suministra el sustrato a estos microorganismos (Leeson E Summers, 2000; ICONTEC, 1994).

Es así, como se debe explorar alternativas con otras materias primas, como la melaza de caña, que contiene componentes esenciales que favorecen el crecimiento de las BAL y proporcionan alternativas de relación costo-beneficio, a nivel de producción y de rentabilidad, incrementando la proliferación del microorganismo rápidamente, valiéndose de sustratos disponibles para su uso; aunque pueden existir otros sustratos más económicos, un primer acercamiento a evaluar las condiciones favorables para el incremento de $L$. plantarum en melaza de caña puede generar nuevas iniciativas de alcance para producción (Vargas et al. 2004; Ortiz et al. 2008).

El objetivo principal de este estudio fue evaluar las condiciones óptimas, bajo las circunstancias de la investigación actual, de crecimiento de L. plantarum en melaza de caña, como sustrato.

\section{MATERIALES Y MÉTODOS}

Conservación de la cepa: La cepa de Lactobacillus plantarum WS417, donada por el Instituto Zentralinstitut für Emährungs und Lebensmittelforschung (Ziel), de la Universidad München, Freising (Alemania), fue sembrada en agar Man Rogosa y Sharpe (MRS) (Sharlau, España) e incubada a $30^{\circ} \mathrm{C} \pm 0,1$, por 48 horas sin agitación y en aerobiosis. La cepa fue conservada en tubos inclinados de MRS a $4 \pm 1^{\circ} \mathrm{C}$ y en caldo MRS, con $30 \%$ de glicerol $\mathrm{a}-80^{\circ} \mathrm{C}$.
Evaluación del crecimiento microbiano a diferentes concentraciones de sustrato: Según el protocolo con modificaciones de Ortiz et al. (2008), se utilizó un Erlenmeyer de $250 \mathrm{~mL}$ con $50 \mathrm{~mL}$ de melaza estéril a diferentes concentraciones ( $5 \%, 10 \%, 20 \%, 25 \%$ y $30 \%$ p/v) $\mathrm{pH} 5,2+/-0,1$, los cuales, fueron inoculados con $1 \mathrm{~mL}$ de un cultivo overnight de $L$. plantarum WS417 e incubados a $30 \pm 1^{\circ} \mathrm{C}$ a $100 \mathrm{rpm}$, durante 24 horas. La concentración inicial del inoculo en los erlenmeyer fue de $10^{2}$ UFC/ $\mathrm{mL}$. Para cada ensayo, el crecimiento fue determinada realizando recuentos de Unidades Formadoras de Colonia (UFC) en superficie, a las 48 horas.

Evaluación del crecimiento a diferentes $\mathrm{pH}$ : Para cada concentración de melaza (20\% y $25 \%, 30 \%$, p/v), se ajustó el pH de los erlenmeyer a 5,2 $\pm 0,1,6,0 \pm 0,1$ con $\mathrm{KOH}$ y $\mathrm{HCl}$ al $40 \%$, los cuales, fueron inoculados con $1 \mathrm{~mL}$ de un cultivo overnight de L. plantarum WS417 $\left(10^{2} \mathrm{UFC} / \mathrm{mL}\right)$ en cada tratamiento. Se incubaron a $30 \pm 1^{\circ} \mathrm{C}$ a $100 \mathrm{rpm}$ durante 24 horas y se determinó el crecimiento, por medio de recuentos en placa.

Evaluación del crecimiento a diferentes temperaturas: El crecimiento fue evaluado mediante recuentos celulares en placa, sometiendo los tratamientos (diferentes concentraciones de sustrato a diferentes $\mathrm{pH}$ ), a temperaturas de $25 \pm 1^{\circ} \mathrm{C}, 30 \pm 1^{\circ} \mathrm{C}$ y $35 \pm 1^{\circ} \mathrm{C}$, por 24 horas, en agitación constante (100rpm).

Análisis estadísticos: Cada uno de los ensayos fue realizado por duplicado (Tabla1). Los datos se analizaron usando modelos lineales, como el análisis de varianza factorial, con el programa de Statistix 8,0 (1985-2003 Analytical Software), para observar interacciones de las variables respecto al recuento microbiológico en placa.

\section{RESULTADOS Y DISCUSIÓN}

Crecimiento en diferentes concentraciones de sustrato: Respecto al crecimiento evaluado con diferentes concentraciones, se observó que el crecimiento de $L$. plantarum fue de $10^{9}$ en $20 \%$ y $25 \%$ y se obtuvo recuentos más bajos de $10^{6}$ y $10^{7}$, con las demás concentraciones $(5 \%, 10 \%, 30 \%)$. Se debe tener en cuenta que la concentración inicial del inoculo estaba en $10^{2} \mathrm{UFC} / \mathrm{mL}$. Los recuentos finales de los tratamientos al ser incubados presentaban exponentes de $10^{9}$, lo que indica, que se incrementaba siete unidades el crecimiento bacteriano, en 24 horas, inoculando la melaza de caña al $20 \%$ y $25 \%$ (Tabla 1, Figura 1 y 2). 
Tabla 1. Tratamientos utilizados para evaluar concentraciones de sustrato (melaza de caña), $\mathrm{pH}$ inicial de la melaza $(5,2 \pm 0,1)$, temperatura $\left(30^{\circ} \mathrm{C}\right)$ y tiempo de incubación (24h) y agitación (100rpm).

\begin{tabular}{|c|c|c|c|c|}
\hline Tratamientos & $\mathrm{pH}$ inicial & $\begin{array}{c}\text { Temperatura } \\
{ }^{\circ} \mathbf{C}\end{array}$ & $\begin{array}{c}\text { Concentración sustrato } \\
\% \mathrm{p} / \mathbf{v}\end{array}$ & Tiempo (horas) \\
\hline $\mathbf{1}$ & 5,32 & $30 \pm 1$ & 20 & 24 \\
\hline $\mathbf{2}$ & 4,52 & $30 \pm 1$ & 20 & 24 \\
\hline $\mathbf{3}$ & 5,31 & $25 \pm 1$ & 20 & 24 \\
\hline $\mathbf{4}$ & 4,48 & $25 \pm 1$ & 20 & 24 \\
\hline $\mathbf{5}$ & 5,30 & $30 \pm 1$ & 25 & 24 \\
\hline $\mathbf{6}$ & 4,49 & $30 \pm 1$ & 25 & 24 \\
\hline $\mathbf{7}$ & 5,31 & $25 \pm 1$ & 25 & 24 \\
\hline $\mathbf{8}$ & 4,50 & $25 \pm 1$ & 25 & 24 \\
\hline $\mathbf{9}$ & 5,20 & $30 \pm 1$ & 30 & 24 \\
\hline $\mathbf{1 0}$ & 4,49 & $30 \pm 1$ & 30 & 24 \\
\hline
\end{tabular}

Lo anterior puede ser atribuido a la transformación de la sacarosa a monómeros de azúcar (glucosa y fructosa) por la enzima invertasa, que puede disminuir su actividad a concentraciones altas de sustrato, a determinadas temperaturas $\mathrm{y} \mathrm{H}$, permitiendo el aumento de la velocidad de crecimiento (Kazuhiko \& Kozo, 1995). En contraste, se obtuvo que a bajas concentraciones de melaza $(5 \%$, $10 \%$ ) hay una deficiencia de carbono, que se refleja en la disminución de los recuentos (Ortiz et al. 2008).
A concentraciones de melaza más elevadas (30\%), las bacterias alcanzan la fase estacionaria más rápido, antes de consumir todo el sustrato, lo que hace que el microorganismo no produzca más biomasa, por el estado de saturación del sustrato, respecto a la concentración microbiana.

Crecimiento a diferentes $\mathrm{pH}$. Se demostró que empleando un $\mathrm{pH}$ de 4,5 $\pm 0,1$ y 6,0 $\pm 0,1$, los recuentos

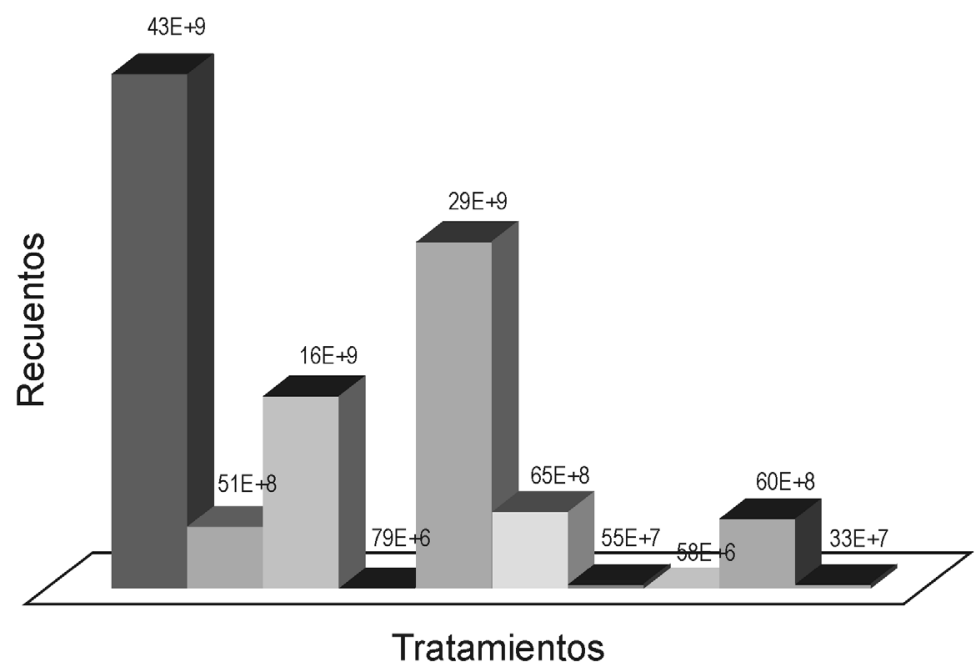

Figura 1. Efecto de los tratamientos sobre el recuento directo en placa de agar MRS. 


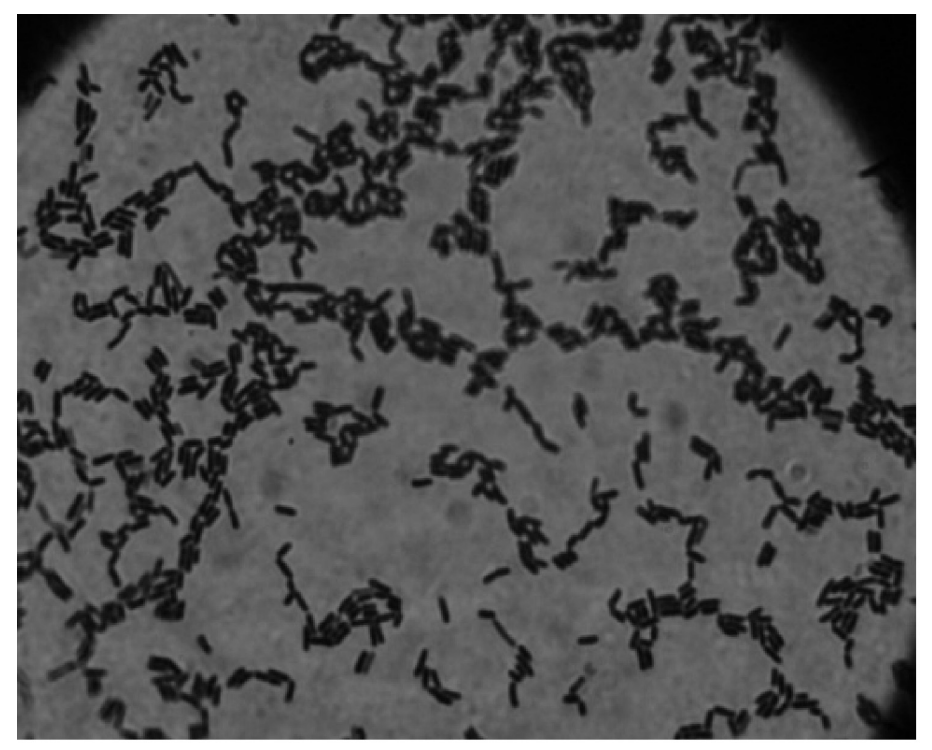

a

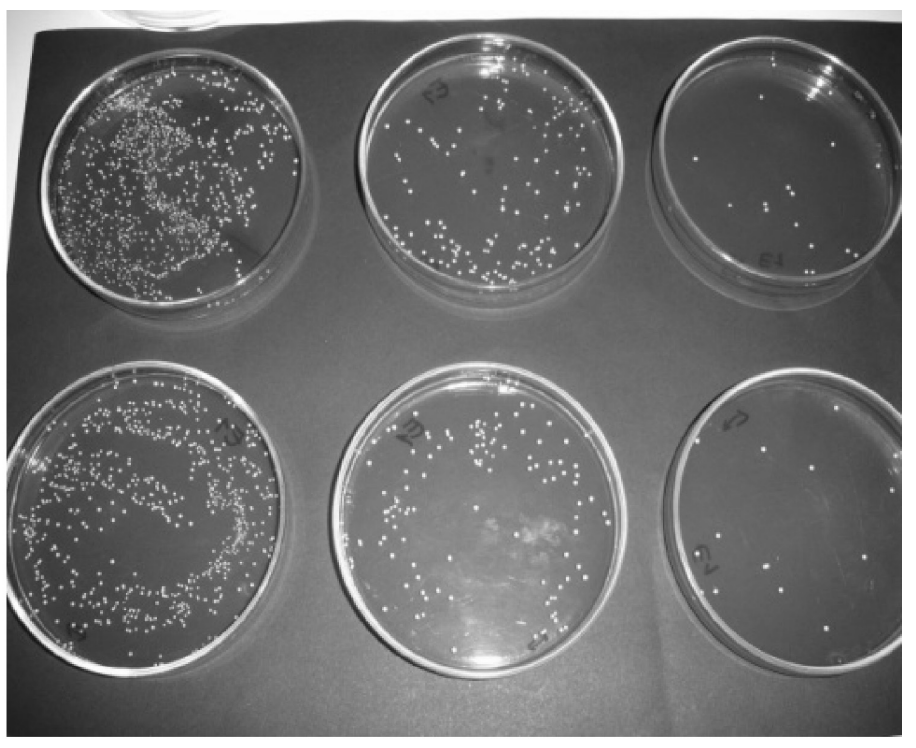

b

Figura 2. a) Lactobacillus plantarum con tinción de Gram, donde se observan bacilos cortos Gram positivos, posterior a la inoculación de los Erlenmeyer con melaza, además de la confirmación y pureza del cultivo, determinada por la apariencia microscópica. b) recuentos (48h) en placa en superficie en agar MRS de L. plantarum, en tratamiento de $30 \pm 1^{\circ} \mathrm{C}, 20 \%, 100 \mathrm{rpm}$, pH inicial 5,2, 24 horas post-inoculación en el Erlenmeyer, en diluciones $10^{7}, 10^{8}, 10^{9}$.

se disminuían respecto a un pH inicial de 5,2 \pm 1 (pH inicial de la melaza), entre una o dos unidades logarítmicas, dependiendo de la concentración del sustrato. La agitación demostró mejorar el crecimiento bacteriano respecto a tratamientos que se evaluaron sin exponer los erlenmeyer en shaker termostatado (Ortiz et al. 2008).

Por otro lado, se midió el pH final de los Erlenmeyer inoculadas de los diferentes tratamientos y se observó una disminución de, aproximadamente, una unidad en el rango de acidez, presentando valores del $\mathrm{pH}$ final en $4 \pm 0,1$, cuando se trabajaba con pH inicial de $5,0 \pm 0,1$, lo cual, evidenció la actividad metabólica de la bacteria.

Las bacterias ácido lácticas crecen adecuadamente bajo condiciones microaerófilicas con cantidades de oxígeno, entre un 2 y $10 \%$; además, en $\mathrm{pH}$, donde los medios son ligeramente ácidos, en un rango de 4,5 a 6,4, bajo condiciones de temperatura entre 30 y $40^{\circ} \mathrm{C}$. Cuando un medio de cultivo alcanza la alcalinidad o neutralidad, el crecimiento de las bacterias tiende a disminuir (Ortiz et al. 2008).

Por otro lado, la agitación incrementó la velocidad de transferencia de nutrientes del medio a las células, mejoró la homogenización de nutrientes y aumentó la velocidad de transferencia de productos metabólicos de las células hacia el medio. Se registró un aumento en el recuento de tres unidades logarítmicas, cuando los tratamientos eran sometidos a agitación constante $\left(10^{9}\right)$, respecto a los que no recibieron el tratamiento $\left(10^{6}\right)$.

Crecimiento a diferentes temperaturas: En placa para la determinación del crecimiento en los diferentes tratamientos mostraron que a temperaturas de $25 \pm 1^{\circ} \mathrm{C}$ y $35 \pm 1^{\circ} \mathrm{C}$, los recuentos de las BAL fueron menores que a $30 \pm 1^{\circ} \mathrm{C}$, en cualquiera de las condiciones evaluadas. El consumo de los sustratos de la melaza, como son azúcares, fósforo, nitratos, se consumen más rápido a temperaturas altas, pero se evidencia incremento de los recuentos a temperaturas menores $\left(30 \pm 1^{\circ} \mathrm{C}\right)$ (Ortiz et al. 2008).

Por lo anterior, se podría afirmar que se lleva a cabo el proceso conocido como inversión de la sacarosa; la presencia de esta enzima depende de las concentraciones de glucosa, de la temperatura de $30 \pm 1^{\circ} \mathrm{C}$, donde se evidencia mayor actividad de la enzima invertasa (Ortiz et al. 2008), que está directamente relacionada con la 
actividad metabólica, debido a que la enzima se encuentra cerca de la superficie de la célula y puede actuar mejor a estas condiciones (Zech $\mathcal{E}$ Gorish, 1995).

Análisis estadístico: En promedio, el crecimiento de L. plantarum evaluado con melaza de caña al $20 \%$ fue mayor que en la del 5\% y 10\%. Además, se evidenció que no existe diferencia estadísticamente significativa $(p>0,05)$ en el recuento promedio, evaluado con las concentraciones de melaza del 20 y $30 \%$. Por lo tanto, se seleccionó la concentración de melaza de caña del $20 \%(\mathrm{p} / \mathrm{v})$, que permite la reducción de costos y el crecimiento óptimo del microorganismo (Ortiz et al. 2008). El tratamiento al $20 \%, 30 \pm 1^{\circ} \mathrm{C}$ de incubación durante 24 horas, rango de $\mathrm{pH}$ de 5,2 $\pm 0,1$ y 100rpm, obtuvo un recuento de $43 \times 10^{9} \mathrm{UFC} / \mathrm{mL}$.

Se observó una relación entre el aumento de la concentración de melaza con el recuento, por su valor beta positivo; sin embargo, la variación no es estadísticamente significativa por un valor $p=0,082$, resultado que concuerda con estudios previos, según Ortiz et al. (2008), en donde han utilizado esta concentración para el incremento de levaduras, ya que en sustratos del $30 \%$ o más, la cantidad de sólidos disueltos contribuyen con el estrés osmótico de las células en cultivo.

Por otro lado, para los microorganismos, el crecimiento es la respuesta principal a las condiciones fisico-químicas del medio en el que se encuentran, de tal forma que el crecimiento es el resultado, tanto de la replicación como de los cambios, en el tamaño de la célula microbiana, debido a la extracción de los nutrientes proveídos por el medio y, posteriormente, convertidos en compuestos biológicos, usados para la producción de energía y para la síntesis o la formación de productos. Cuando se transfiere el inoculo al medio de fermentación, los microorganismos toman los nutrientes necesarios para su metabolismo y los transforman en biomasa, aunque este proceso puede durar de minutos a horas. Se debe tener en cuenta que diversos factores, como la temperatura, el $\mathrm{pH}$ y la concentración de sustrato, afectan significativamente los patrones de crecimiento y la formación de productos asociados con el metabolismo del microorganismo, por eso, es necesario que el sustrato a evaluar sea uno de los factores determinantes al realizar un estudio comparativo de diferentes condiciones.
Finalmente, en la industria láctea y cárnica colombiana podría existir una gran aceptación de nuevas alternativas y propuestas para el crecimiento de bacterias ácido lácticas de uso comercial, debido a que se incrementaría el rendimiento y la calidad de la producción animal, la prevención de enfermedades y el uso indiscriminado de antibióticos y se mejoraría el aprovechamiento de los recursos agrícolas y la tecnología disponibles en el país; además, el contenido nutricional de alimentos de consumo masivo estarían mejor suplementados con cepas probióticas, se ampliaría la gama de aplicaciones de los probióticos a otros alimentos, ya sea para consumo humano o animal y, finalmente, sería benéfico para el consumidor, ya que los valores de los productos se disminuirían por el ahorro en los costos de importación de los probióticos (Vargas et al. 2004).

Se concluye, que esta investigación permitió evaluar algunos aspectos relacionados con las condiciones óptimas de crecimiento de $L$. plantarum, en las condiciones del estudio, utilizando un subproducto de la industria azucarera, la melaza de caña, con la cual, se logró incrementar el desarrollo microbiano de bacterias ácido lácticas, a partir de medios no lácticos. Los resultados hallados indican que el tratamiento que permitió el máximo crecimiento del microorganismo e incrementó los recuentos siete unidades logarítmicas a las 24 horas fue empleando sustrato al $20 \%$. Se recomienda la melaza de caña, como sustrato, para realizar más investigaciones a nivel de laboratorio y, posteriormente, poseer herramientas de acercamiento a planta piloto, para lograr proponer este sustrato, como un medio de trabajo pertinente, que optimice la propagación de las bacterias ácido lácticas a nivel industrial.

AGRADECIMIENTOS: Los autores agradecen al Comité de Investigación de la Facultad de Ciencias Biológicas y al Laboratorio de Ecología Microbiana y de Alimentos LEMA, de la Universidad de Los Andes, Bogotá, Colombia, por el apoyo y financiación para la realización de esta investigación. Conflictos de intereses: El manuscrito fue preparado y revisado con la participación de todos los autores, quienes declaramos que no existe ningún conflicto de intereses que ponga en riesgo la validez de los resultados presentados. Financiación: Este estudio fue financiado por la Universidad de Los Andes, Comité de Investigación de la Facultad de Ciencias Biológicas, Laboratorio de Ecología Microbiana y de Alimentos LEMA. 


\section{BIBLIOGRAFÍA}

1. ALPINA. Disponible desde Internet en: www.alpina. com.co (con acceso 04/02/10).

2. CABEZA, H.E.A. 2006. Bacterias ácido lácticas (BAL): aplicaciones como cultivos estárter para la industria láctea y cárnica. 1-12. (Colombia). Disponible desde Internet en http://enalcahe.googlepages. com/Bacteriascido-lcticas_BAL__aplicacio.pdf (con acceso 04/02/10).

3. CASTELLANO, P.; BELFIORE, C.; FADDA, S.; VIGNOLO, G. 2008. A review of bacteriocinogenic lactic acid bacteria used as bioprotective cultures in fresh meat produced in Argentina. Meat Sci. 79(3):483-499.

4. CASTRO, M.; RODRÍGUEZ, F. 2005. Levaduras: probióticos y prebióticos que mejoran la producción animal. Rev. Corpoica. (Colombia) 6(1):1-27.

5. COGAN, T.; BARBOSA, M.; BEUVIER, E.; BIANCHISALVADORI, B.; COCCONCELLI, P.; FERNANDES, I.; GÓMEZ, J. 1997. Characterization of the lactic acid bacteria in artisanal dairy products. J. Dairy Res. 64:409-421.

6. DANE. 2006. Observatorio Agrocadenas Segundo informe de coyuntura de 2006. p.1-35 (Colombia). Disponible desde internet en: http://www.redlactea. org/documentos/Inf\%20coy\%202\%202006.pdf (con acceso 04/02/10).

7. FIORENTINI ÂNGELA, M.; SANT'ANNA ER-NANI, S.; PORTO ANNA, C.S.; MAZO JACIARA, Z.; FRANCO BERNADETTE, D.G.M. 2001. Influence of bacteriocins produced by Lactobacillus plantarum in the shelf-life of refrigerated bovine meat. Brazilian J. Microbiol. 32:42-46.

8. GÁLVEZ, A.; ABRIOUEL, H.; LUCAS LÓPEZ, R.; BEN OMAR, N. 2007. Bacteriocin based strategies for food biopreservation. Internal. J. Food Microbiol. 120:51-70.

9. HOLZAPFEL, W. 2001. Introduction to pre and probiotic. Food Res. Internal. p.963-969.
10. HONIG, P. 1974. Principios de Tecnología Azucarera. $2^{a}$ ed. Compañía Edit. Continental. (México). p.23-54.

11. INSTITUTO COLOMBIANO DE NORMAS TÉCNICAS ICONTEC. 1994. Industrias alimentarias e industriales de bebidas. Melaza de caña NTC.587.

12. KAZUHIKO, T.; KOZO, T. 1995. Factors affecting the ethanol productivity of yeast in molasses. $\mathrm{J}$. Ferment. Bioeng. 79(5):449-452.

13. LEE, Y.J.; SALMINEN, S. 1995. The coming age of probiotic. Trends in Food Sci. Techn. 6:241-245.

14. LEESON, S.; SUMMERS, J. 2000. Nutrición Aviar Comercial. Editorial Le’Print Club Express Ltda. (Colombia). p.43-45.

15. MANTELLO, R.S. 2007. Materias primas: Yogurt: El yogurt y los alimentos probióticos. (Argentina). Disponible desde internet en: http://www.mundohelado.com/materiasprimas/yogurt/yogurt12.htm (con acceso 04/02/10).

16. ORTIZ, A.; REUTO, J.; FAJARDO, E.; SARMIENTO, S.; AGUIRRE, A.; ARBELÁEZ, G.; GÓMEZ, D.; QUEVEDO, H.B. 2008. Evaluación de la capacidad probiótica "in vitro" de una cepa nativa de Saccharomyces cerevisiae. Universitas Scientiarum. (Colombia). 13(2):138-148.

17. SHAH, N.P., LANKAPUTHRA, E.V. 2002. Improving viability of Lactobacillus acidophilus and Bifidobacterium spp. in yogurt. Internal Dairy J. 7:349-356.

18. SIMOVA, E.; BESHKOVA, D.; ANGELOV, A. 2002. Lactic acid bacteria and yeasts in kefir grains and kefir made from them. J. Indust. Microbiol. $\varepsilon$ Biotechn. 28:1-6.

19. SILVEIRA RODRÍGUEZ, M.B.; MONEREO MEGIAS, S.; MOLINA BAENA, B. 2003. Alimentos funcionales y nutrición óptima. ¿Cerca o lejos? Rev. Española de Salud Pública. 77(3):317-331.

20. STATISTIX 8,0. Analytical Software (1985-2003). 
21. SWAN, H.; KARALAZOS, A. 1990. Las melazas y sus derivados. Rev. Tecn. Geplacea. (España).19:7882.

22. VARGAS, E.M.; GÓMEZ, J.C.; PARRA, M.E.; ROMERO, M.A. 2004. Obtención de Microorganismos Probióticos en un medio no láctico. 166-175 (Colombia). Disponible desde internet en: http:// revistaing.uniandes.edu.co/pdf/Rev1917.pdf?ri $=821 \mathrm{~d} 8 \mathrm{c} 5 \mathrm{~b} 0 \mathrm{c} 8193719707 \mathrm{a} 9 \mathrm{a} 8131$ daacc $(\mathrm{con}$ acceso 04/02/10).
23. VÁSQUEZ, M.S.M.; SUÁREZ, M.H.; ZAPATA, B.S. 2009. Utilización de sustancias antimicrobianas producidas por bacterias ácido lácticas en la conservación de la carne. Rev. Chil. Nutr. 36(1):6471.

24. ZECH, M.; GORISCH, H. 1995. Invertase from Saccharomyces cerevisiae: Reversible inactivation by component of industrial molasses media. Enzyme Microbial and Technology. 17:41-46

Recibido: Marzo 26 de 2009

Aceptado: Marzo 8 de 2010 